\title{
PROCESSING AND MODEL DESIGN OF THE GAMMA OSCILLATION ACTIVITY BASED ON FITZHUGH-NAGUMO MODEL AND ITS INTERACTION WITH SLOW RHYTHMS IN THE BRAIN
}

\author{
Evgeniia S. Sevasteeva \\ Saint Petersburg State University \\ Institute for Problems \\ of Mechanical Engineering \\ Saint-Petersburg, Russia \\ esevasteeva@outlook.com
}

\author{
Sergei A. Plotnikov \\ Saint Petersburg State University \\ Institute for Problems \\ of Mechanical Engineering \\ Saint-Petersburg, Russia \\ waterwalf@gmail.com
}

\author{
Volodymyr Lynnyk \\ The Czech Academy of Sciences, \\ Institute of Information Theory \\ and Automation \\ Prague, Czech Republic \\ voldemar@utia.cas.cz
}

Article history:

Received 15.12.2021, Accepted 27.12.2021

\begin{abstract}
The brain is in a state of processing information 24 hours a day. There are millions of processes proceeding in it accompanied by various spectra of rhythms. This paper tests the hypothesis that the slow delta rhythm excites the gamma rhythm oscillations. Unlike other papers, we determine the slow rhythm spectrum not at the hypothesis stage but during the experiment. We design algorithms of filtration, envelope extraction, and correlation coefficient calculation for signal processing. Moreover, we examine the data on all electroencephalogram channels, which allows us to make a more reasonable conclusion. We confirm that a slow delta rhythm excites a fast gamma rhythm with an amplitude-phase type of interaction and calculate a delay between these two signals equal to about half a second.
\end{abstract}

\section{Key words}

Gamma rhythm, Correlation, Oscillation, Electrocorticogram, FitzHugh-Nagumo model.

\section{Introduction}

The human brain processes information almost continuously throughout life. In [Neske, 2016], the authors noted that the cerebral cortex and thalamus exhibit quite diverse and rich activity even during the most inactive periods of human life. This activity manifests itself in slow (up to $1 \mathrm{~Hz}$ ) neural oscillations. There are confirmations that these oscillations are generated by vast ensembles of synchronized neurons working as a whole. Such an organization provides the possibility of such complex cognitive processes as memory, thinking, and decision-making [Papadimitriou et al., 2020; Buzsáki, 2010; Folschweiller and Sauer, 2021].

The interaction between its various areas provides the brain work consistency through neural oscillations, i.e. electrical activities of different frequencies. There is a generally accepted classification of brain ranges, which includes high-frequency bands (alpha $(8-12 \mathrm{~Hz})$, beta $(12-30 \mathrm{~Hz})$, gamma $(30-150 \mathrm{~Hz}))$, and low-frequency (theta $(4-8 \mathrm{~Hz})$ and delta $(0-4 \mathrm{~Hz})$ ). Gamma rhythms, in turn, are divided into rough $(30-80 \mathrm{~Hz})$ and high gamma $(80-150 \mathrm{~Hz})$ signals [Moran and Hong, 2011; Gonzalez Burgos et al., 2015]. However, the latter is usually very noisy and does not lend itself very well to analysis. There are enough studies that reveal the independent activity of different rhythms in different situations [Carmichael et al., 2017; Clayton et al., 2018; Ghosn et al., 2020]. Moreover, studies are suggesting a direct coupling between rhythms bands [Chuderski, 2016; Canolty and Knight, 2010; Abubaker et al., 2021]. Also, there are works revealing the connection between various human states and deviations in the brain rhythms. For instance, abnormalities as post-stroke effects [Kiselev et al., 2019] or as a consequence of the aging [Frolov and Pitsik, 2021]. Rhythms diversity could be provided even by the human personality traits and mental abilities while doing some tasks [Pisarchik et al., 2018]. Thereby, we decide to research works studied when the coupling deviations manifest themselves.

While investigating research on the rhythms coupling, we were particularly interested and inspired by [Moran and Hong, 2011]. It reveals the concept of synchronous deviations in the slow and fast bands in schizophrenia: against the background of slow rhythms anomalies, anomalies in gamma rhythm appeared. One more 


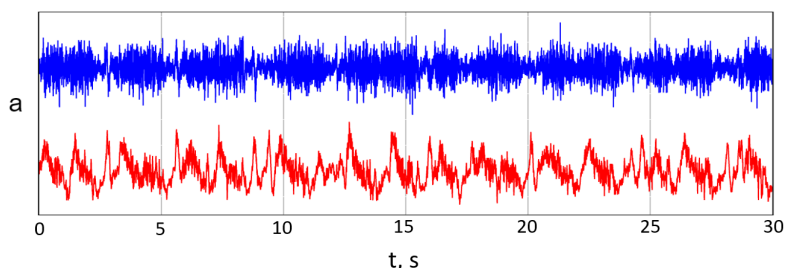

$t, s$

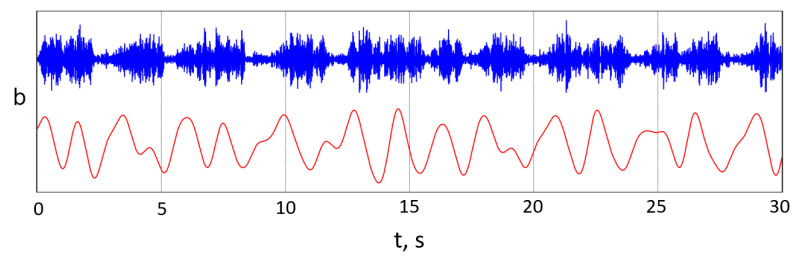

Figure 1. a: Unfiltered fragment of the ECoG recordings. The highresistance electrode recording is indicated by the blue color, whereas the red one indicates the low-resistance electrode recording. b: Filtered fragment of the ECoG recordings. The high-frequency gamma rhythm $x(t)$ is represented by blue, whereas red represents the low-frequency signal $y(t)$

work about that conducting a bit later also interested us in [Gonzalez Burgos et al., 2015].

Nevertheless, how the fast and slow processes in the brain interact is still a mystery. This interaction can be accomplished in a variety of ways [Buzsáki and Wang, 2012; Osipova et al., 2008], including amplitude correlations [Bruns and Eckhorn, 2004; Mohamed et al., 2004], phase to phase synchronization [Sauseng et al., 2019; Palva, 2005; Li et al., 2018], phase to frequency principle [Jensen and Colgin, 2007] and phase to power locking [Park et al., 2016; Köster et al., 2018; Berman et al., 2015].

The power-to-power link has a weaker cross-frequency correlation [Bruns and Eckhorn, 2004] and is commonly thought of in the context of a single rhythm [Buzsáki and Wang, 2012]. The problem of zero-lag phase synchronization in phase to phase synchronization exists [Fell and Axmacher, 2011]. The phase-to-power relationship is particularly relevant since a low-frequency signal (e.g. delta, theta, or alpha) can generate the highfrequency rhythm (e.g. gamma). This knowledge can be exploited with biological neuron models to simulate an excitable system that produces high-frequency oscillations. This paper continues the research presented at the DCNA 2021 conference [Sevasteeva and Plotnikov, 2021].

The rest of this paper is organized as follows: In Sec. 3, the signal processing tool is developed: Subsec. 3.1 considers the filtering system construction, while Subsec. 3.2 deals with envelope extracting algorithms. Then in Sec. 4, the data analysis is performed: time delays between considered signals are evaluated and the experiment results are presented. Section 5 presents the results of the simulation of gamma rhythm using the modified FitzHugh-Nagumo (FHN) model. Finally, the conclusions are drawn in Sec. 6.

\section{Data description}

The simple anesthetized. Wistar rat ECoG was recorded during acute experiments. Low-resistance electrode recordings typically contain predominantly slow dynamics and essentially no high-frequency rhythms, because high-frequency signals are restricted by tiny areas, but low-frequency signals need synchronous activity across wide areas of the brain (see [Furth et al., 2013]). The gamma rhythm was recorded using the Neuronexus E32 - 600 - 10 - 100 multi-electrode array with 32 registration sites of $100 \mu \mathrm{m}$ each (site impedance is $500 \mathrm{k} \Omega$ ) and cross-site $600 \mu \mathrm{m}$ intervals. These features allow for the recording of a stable gamma rhythm $(30-80 \mathrm{~Hz})$ on a small scale with sufficient localization, even under general anesthesia.

This multi-electrode array was implanted in the left hemisphere, near the sensory cortex. Gold plated screw electrodes (impedance $25-50 \mathrm{k} \Omega$ ) were utilized to gather the low-frequency signal. Over the cerebellum, one of these screws was employed as an indifferent electrode. Low-resistance electrodes, as expected, produced a sluggish rhythm during anesthesia, i.e., the characteristic low-frequency oscillations seen during sleep and anesthesia.

On three rats, three trials with a total duration of 128 seconds and a sampling rate of $2 \mathrm{kHz}$ were conducted. At this work, we were considering an experiment taken from only one rat, since biologists decided that two of the three experiments were not very successful and noise got into the data.

\section{Tools development}

This section examines the various existing approaches, taking into consideration their flaws. It proposes a universal instrument for signal filtering and envelope extraction. Now let us illustrate experimental data to better understand these techniques. It is a 128-s. electrocorticogram (ECoG) with thirty-two channels: twentyeight from high-resistance electrodes and four from lowresistance electrodes. A sample rate of $2 \mathrm{kHz}$ is provided. In Fig. 1a, an unfiltered segment of the ECoG recordings is shown. The blue color indicates the highresistance electrode recording, whereas the red one indicates the low-resistance electrode recording. The highresistance electrode recording will be utilized to obtain a gamma rhythm, while the low-resistance electrode data will be used to get a slow delta rhythm.

\subsection{Filtering}

The initial stage in the analysis is to identify the signals that fall within a suitable frequency range. We consider Filters in both FIR and IIR forms. The first type of filter was later abandoned because the order of such filters is too high to provide the necessary band characteristics [Mitra, 2001]. As a result, [Rabiner, 1975] was chosen as the IIR filter having the cleanest amplitude-frequency 
response at pass-band frequencies. The amplitude responses of several IIR-filters are shown in Fig. 2a. A zero-shift filter was implemented [Mitra, 2001] to prevent producing nonlinear phase shifts that are crucial in ECoG signal processing while making Butterworth filter amplitude response sharper. In Fig. 2b, the amplitudefrequency responses of the Butterworth and zero-phase filters are demonstrated. In contrast to the first one, the second filter has no nonlinear phase shifts and has more stark amplitude responses, as shown in this diagram.
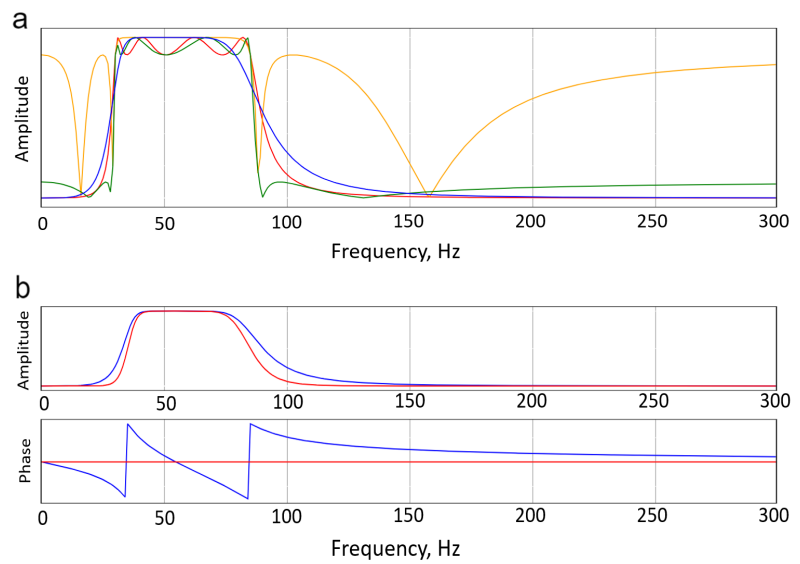

Figure 2. a: The band-pass Chebyshev type I (red), elliptic (green), and Butterworth (blue) filter amplitude responses. The order of the filters is equal to four. 35 and $85 \mathrm{~Hz}$ are the bandwidth restrictions. b: The band-pass Butterworth (blue) and zero-phase (red) filter amplitude-frequency responses. The order of the filters is equal to four. 30 and $85 \mathrm{~Hz}$ are the bandwidth bounds.

The recording of the high-resistance electrode is digitally band-pass-filtered in the frequency domain between 30 and $85 \mathrm{~Hz}$ to obtain a gamma rhythm. This filtered fast-frequency signal will be denoted as $x(t)$. One can see in Fig. 1b that there are periods of gamma activation and extinction of approximately $1 \mathrm{~s}$. duration, i.e. the whole period approximately equals $2 \mathrm{~s}$. Hence, we choose the frequency domain for the low-resistance electrode recording between 0 and $0.5 \mathrm{~Hz}$, i.e. identify the slow exciting signal as a delta rhythm, to show the correlation between this low-frequency signal and gamma activation and extinction. We denote this filtered lowfrequency signal as $y(t)$. It can also be noticed that there is a connection between $x(t)$ and $y(t)$ : the first one activates when the second one is above zero and extinct else. To find the numerical measure of two signal interdependence, we should somehow transform the highfrequency signal $x(t)$.

To receive a gamma rhythm, the recording of the highresistance electrode is digitally band-pass-filtered in the frequency domain between 30 and $85 \mathrm{~Hz} . x(t)$ is the notation for the filtered fast-frequency signal. There are intervals of gamma activation and extinction of roughly 1 s. duration in Fig. 1b, i.e. the entire period is approxi- mately $2 \mathrm{~s}$. To demonstrate the association between this low-frequency signal and gamma activation and extinction, we chose a frequency domain for the low-resistance electrode recording between 0 and $1 \mathrm{~Hz}$, i.e. identify the slow exciting signal as a delta rhythm. The notation $y(t)$ denotes this filtered low-frequency signal. It is also worth noting that there is a link between $x(t)$ and $y(t)$ : the first one activates when the second one is above zero, and the latter is extinct otherwise. We must somehow transform the high-frequency signal to determine the numerical measure of two signal dependency. It is defined by oscillation activation and extinction intervals that alternate. Thereby, the upper envelope should be calculated to run around these activation and extinction intervals.

\subsection{Extracting envelope}

We select the frequency band for slow signals between 0 and $1 \mathrm{~Hz}$. It corresponds to the estimated period of bursts and attenuations of the fast signal. It is reasonable to assume that this value should also characterize the envelope of our fast signal.
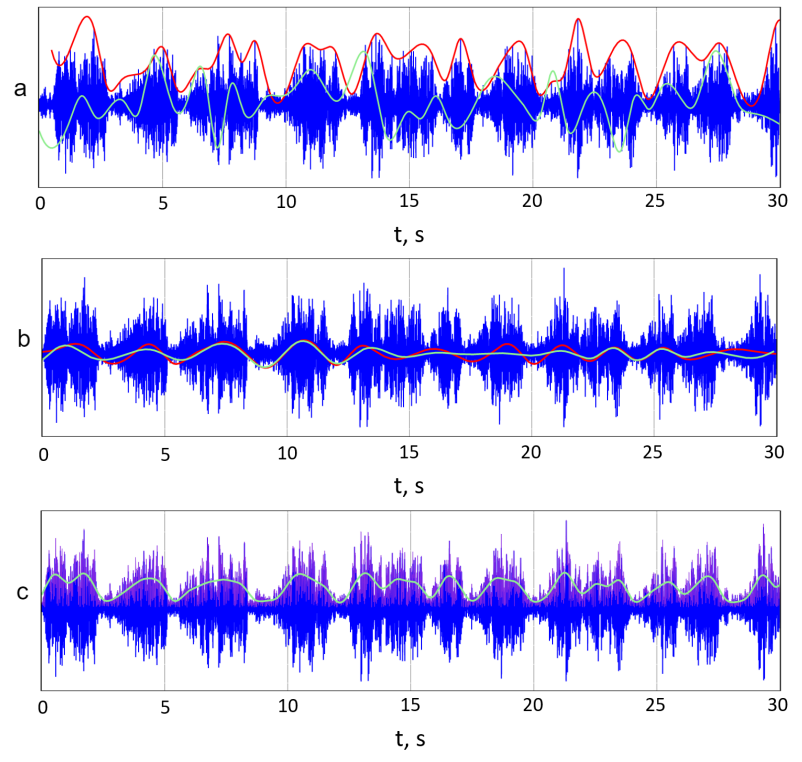

Figure 3. a: Two fast-signal peak envelopes with a peak distance of 1000. The blue color denotes the signal $y(t)$, the green color indicates the envelope with the initial time $t_{0}$, and the red color denotes the envelope with the initial time $t_{0}+0.5 \mathrm{~s}$. b: Two signal $y(t)$ envelopes obtained from the same imf-components of two EMDs. The blue color denotes the signal $y(t)$, the red color denotes the received $i m f$-component for the current interval, and the green color denotes the broader interval. c: $y(t)$ Hilbert transform and filtered version of that transformation. The signal $y(t)$ is denoted by blue, the Hilbert transform envelope of $y(t)$ is denoted by violet, and the filtered Hilbert envelope is denoted by green.

The following methods were initially considered: empirical mode decomposition (EMD) [Gupta, 2019], 
peak-envelope, and Hilbert transform [Panter, 1965] with with some additions. The peak approach necessitates determining the peak-between distance (the frequency at which the peaks will be incorporated as components of the future envelope) and the initial point. Because the gamma rhythm is a high-frequency signal, it is evident that the starting envelope point impacts the final curve. The difference (shift) between two envelope initial points equals $0.5 \mathrm{~s}$. provides completely different results. A demonstration of this fact can be seen in Fig. 3a. As a result of these findings, we decided to disregard the peak-envelope technique.

EMD methods show promising results. Still, it is necessary to select a suitable number of components (intrinsic mode functions or imf-component) for envelope manually for each experiment concerning the duration of the analyzed signal. Applying this method to two intervals (one containing the other) may give different results at their intersection when considering the same imfcomponent. An illustration of this situation can be seen in Fig. 3b. Therefore, despite EMD advantage in isolating the envelope directly from the experimental signal, we had to abandon this approach due to its nonuniversality. Also, this method is computationally expensive compared to the others. The Hilbert transform also works well and does not need individual customization. However, it provides a too detailed envelope. Since that, the low-pass zero-phase filter was implemented to the Hilbert transform envelope. Denote the envelope of the gamma rhythm by $e(t)$.

EMD approaches produce good results, but only after the manual settings. For each experiment, depending on the duration of the examined signal, it is necessary to select an appropriate number of envelope components (intrinsic mode functions or imf-component). When evaluating the same imf-component, applying this approach to two intervals (one including the other) may yield different results at their intersection. In Fig. 3b, you can see an illustration of this circumstance. As a result, even though EMD had the advantage of separating the envelope directly from the experimental signal, we had to forsake it due to its non-universality. In addition, as compared to the others, this method is computationally expensive. The Hilbert transform is also an effective method and does not require personalization. It does, however, produce an overly comprehensive envelope. Since then, the Hilbert transform envelope was passed through the low-pass zero-phase filter. The envelope of the gamma rhythm is denoted by $e(t)$. The envelope calculation result (obtained with the final approach benefit) is shown in Fig. $3 \mathrm{c}$ and is highlighted in green.

\section{Data Analysis}

This section explores the interconnectedness of fast and slow processes in the brain. As previously stated, several principles of cross-frequency interactions exist, and we use the signal-envelope correlation as an alternate method to detect coupling between gamma and low-frequency cycles in this study. We demonstrate that when the low-frequency signal has a positive phase, faster gamma oscillations occur; when the low-frequency rhythm is in the negative phase, gamma oscillation death appears. Unlike the methodologies in [Bruns and Eckhorn, 2004; Canolty et al., 2006; Osipova et al., 2008], our study not only obtains relatively good correlation values but identifies the frequency of the stimulating slow rhythm via experiment analysis. Also, we analyze the time delay between the slow signal and the gamma signal envelope $e(t)$.

Firstly, we evaluate mean value of the slow rhythm and fast-one envelope:

$$
\bar{y}=\frac{1}{|T|} \sum_{t \in T} y(t), \quad \bar{e}=\frac{1}{|T|} \sum_{t \in T} e(t),
$$

where $T$ is an array of the signal measurements $[0 ; 128]$ s., and $|T|$ is its cardinality, since these signals are discrete; After the mean evaluation, we should center the signals:

$$
y_{c}(t)=y(t)-\bar{y}, \quad e_{c}(t)=e(t)-\bar{e} .
$$

And then, we normalize it to compensate for the signal amplitudes difference :

$y_{n}(t)=\frac{1}{\max _{t \in T}\left|y_{c}(t)\right|} y_{c}(t), \quad e_{n}(t)=\frac{1}{\max _{t \in T}\left|e_{c}(t)\right|} e_{c}(t)$.

So, the values of converted signals now fall into the $[-1 ; 1]$ range. We can now calculate the numerical value of signal interdependence. We utilize the Pearson correlation coefficient for this. This coefficient is a statistical measure of linear correlation between two signals with a value ranging from -1 to 1 . We compute the correlation coefficient for different time delays, assuming that the interaction of rhythms may not occur instantly.

$$
\rho(\tau)=\frac{\sum_{t \in \hat{T}} y_{n}(t+\tau) e_{n}(t)}{\sqrt{\sum_{t \in \hat{T}} y_{n}^{2}(t+\tau) \sum_{t \in \hat{T}} e_{n}^{2}(t)}},
$$

where $\tau$ is a delay, $\hat{T}$ is a subset of $T$, which contains elements of the analyzed time interval $[30 ; 100] \mathrm{s}$. The beginning and the end of the experiment are cut off due to the possible presence of interference during the installation of equipment and the beginning of its removal. A closer study of the graphs shows that our signal fluctuates at $0.5 \mathrm{~Hz}$, i.e. the possible delay $\tau$ belongs to the interval $[-2 ; 2]$ s. ( \pm period of the signal).

The effect of cross-correlation $\rho$ on the delay $\tau$ between the slow and fast signal envelope one can see in Fig. 3. The maximum cross-correlation value is 0.662 , corresponding to a delay of $\tau=-0.456 \mathrm{~s}$. That means 
that the high-frequency gamma rhythm behavior is determined by the phase of the slow signal $0.456 \mathrm{~s}$. ago. Another argument for this statement is that the correlation curve has explicit negative minima for positive $\tau=0.566$, i.e. two researched signals enter the opposite phase after about a half of the slow signal $y(t)$ period. It is worth noting that the period of function $\rho(\tau)$ is around $2 \mathrm{~s}$., which is the same as the period of the delta rhythm $y(t)$.
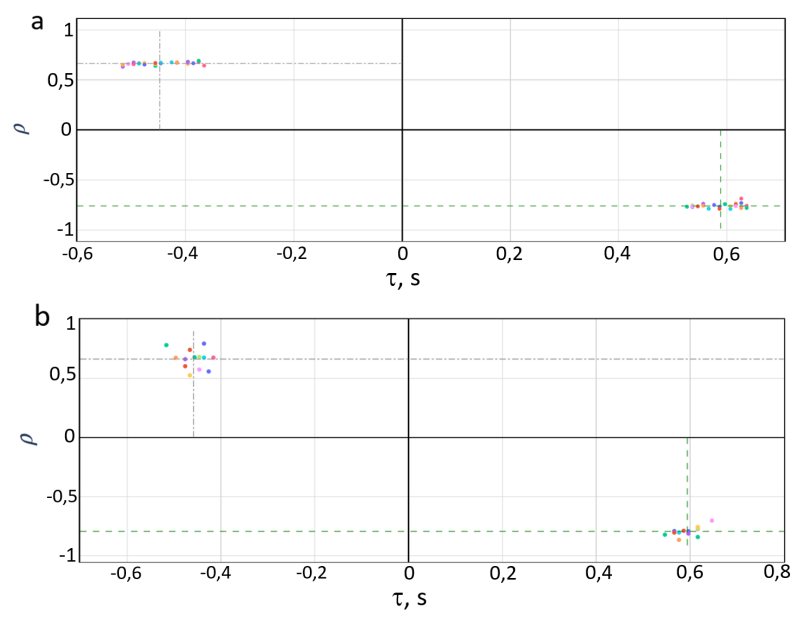

Figure 4. a: Scatter plot of maximum and minimum correlation coefficients and relative time shifts for each channel. Dotted line illustrated average correlation values and time shifts across all channels. b: Scatter plot of correlation values and time shifts for each 20 -s. interval (5-s. increment) across $[30 ; 100]$ s. range.

We receive confirmation of the stated hypothesis but only for the one channel. Now it is logical to make the same steps for other channels and compare what we would take. The pipeline of this step is there: we filter each channel, center and normalize it, evaluate correlation coefficient for $\tau \in[-2 ; 2]$ s., and took the maximum values of the positive and negative correlation (modulo), as well as the corresponding shift times. Then we evaluate the mean correlation coefficient and shift time for positive and negative ones. The result scatter plot one can see in Fig. 4. The dotted lines draw the average correlation values ( 0.66 for positive correlation values, -0.75 for negative values) and time shifts $(-0.446 \mathrm{~s}$. for positive correlation values, $0.586 \mathrm{~s}$. for negative values) across all channels. Even though there are minor differences in time delays for different channels, the envelope correlation with delta signal is greater than 0.62 for each channel. It is worth noticing that these results were received with almost the whole experiment period analysis. Hence, we indicate that our statement is correct. We take the channel with a correlation value more than the average and time shifts nearest to the average shift to the following analysis. We choose the channel based on the positive correlation values because these results are the direct hypothesis confirmation.
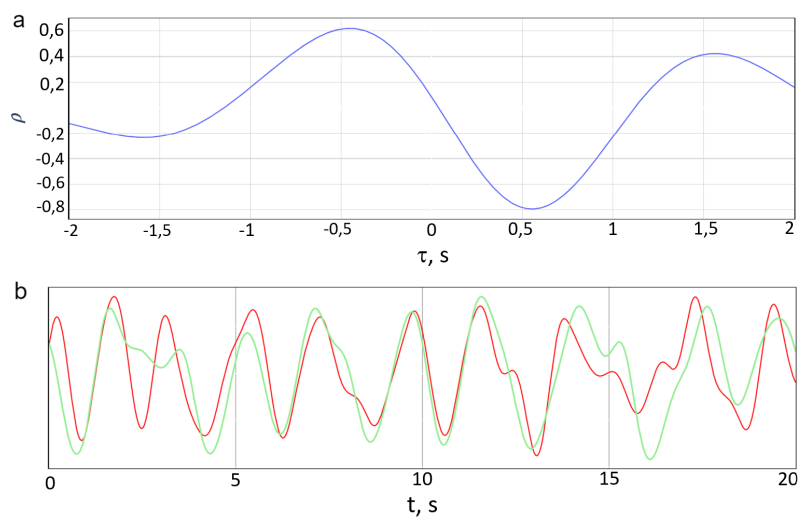

Figure 5. a: Dependence of the cross-correlation $\rho$ between the lowfrequency signal $y_{n}(t+\tau)$ and the high-frequency signal envelope $e_{n}(t)$ on the delay $\tau$ between two signals. b: Dependence of the delayed envelope value $e_{n}(t)$ (green) on the low-frequency signal $y_{n}(t-0.456)$ (red).

After choosing the best channel, we divide our signals from $[30 ; 100]$ s. range into 20 -s. intervals in 5 -s. increments, that is $[30 ; 50]$ s., $[35 ; 55]$ s., ..., $[80 ; 100]$ s., and evaluate correlation within them. We do that because for the next modeling much computation power is needed. And making simulation longer than $20 \mathrm{~s}$. is computationally expensive. The criteria for selecting the interval for analysis are the same for selecting the channel (high correlation, proximity to the average shift). The best result was obtained for the $[35 ; 55] \mathrm{s}$. interval. Correlation has the value $\rho=0.74$ and the time delay equals $\tau=-0.456 \mathrm{~s}$. Figure 5 presents the dynamics of two processed signals, namely centered, normalized and delayed envelope of gamma rhythm $e_{n}(t)$ and centered and normalized delta rhythm $y_{n}(t-0.456)$ during the chosen time interval. One can see that the raising of the envelope value, i.e. the emergence of gamma oscillations, is highly dependent on the increase of the delta rhythm value. The fact that a slow delta signal can excite fast gamma oscillations is thus established.

\section{Modeling}

A single cognitive unit is a closely connected collection of active neurons (cell ensemble), and the spike activity of such a group is the basic unit of neural coding. Oscillators are synchronized clusters of neurons that generate high-frequency oscillations and the gammarhythm is a high-frequency rhythm. By the way, recent studies [Panteley and Loría, 2017] have shown that the neurons cluster oscillations could be described by the same equations as a single neuron with noise addition. To design a model of such oscillators for simulation of gamma rhythm, we modify a simple model of neuron dynamics, namely the FitzHugh-Nagumo (FHN) 
[FitzHugh, 1961; Nagumo et al., 1962] model. Introduce a two dimensional model based on FHN model:

$$
\begin{aligned}
\dot{u}(t) & =\frac{\delta}{\varepsilon}\left[u(t)-\frac{u^{3}(t)}{3}-v(t)+\xi(t)+y(t+\tau)\right], \\
\dot{v}(t) & =\delta[u(t)+a-b v(t)] .
\end{aligned}
$$

where $u$ and $v$ denote an oscillator potential and a recovery variable, respectively. The value for neuron dynamics is $\varepsilon=0.8$, and the time scaling coefficient is $\delta=325$. The value of $\delta$ is chosen to make the system (5) equation oscillate at the same frequency as the gamma rhythm (neural oscillation between 35 and 80 $\mathrm{Hz}) . \quad y(t+\tau)$ is a low-frequency delta rhythm with $\tau=-0.456$, and $\xi(t)$ is a "neuronal" noise, which is supposed to be an unbiased Gaussian white noise, which is a mathematical representation of many natural phenomena. The parameters $a$ and $b$ are constants.

We employ evolutionary algorithms [Libelli and Alba, 2000; Fu et al., 2017] to determine the right values of parameters $a, b$. We fix $b=0.8$ and adjust the value of the threshold $a$ between 0.55 and 1.4 for this reason; the original system (5) has a stable equilibrium point with these values. We simulate the dynamics of the system (5) and determine its normalized and centered envelope $e_{\text {sim }}$ for each value of the threshold $a$. The Pearson correlation coefficient is a statistical measure of linear correlation between high-frequency signal envelope $e_{n}$ and simulated signal envelope $e_{\text {sim }}$ that we employ for this purpose. The maximum correlation value is 0.63 , corresponding to the $a=1.05$ threshold.

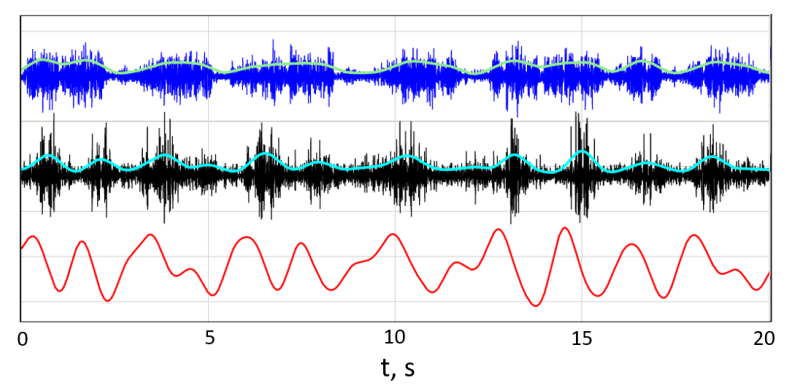

Figure 6. Dynamics of the system (5) potential $u(t)$ and its envelope (indicated by cyan color) in relation to the high-frequency gamma rhythm $x(t)$ and its envelope (marked by blue color) (marked by green color). The low-frequency delta rhythm $y(t)$ is indicated by the red color. $\varepsilon=0.8, \delta=325, a=1.05, b=0.8, \tau=-0.456$ are the system parameters. Zeros are the starting conditions.

The simulation results are shown in Fig. 6. The system (5) dynamics are seen to be dependent on the low-frequency signal $y(t)$ value: when $y(t)$ is high enough, the system exhibits self-sustained periodic firing, whereas when $y(t)$ is low, the system exhibits oscillation death. The firing times of the system (5) coincide with the activation periods of the gamma rhythm $x(t)$. As a result, the FHN model may be used to simulate the gamma rhythm.

\section{Conclusion}

This paper investigated the relationships between fast gamma rhythm and slow brain rhythm. A delta rhythm was discovered as the low-frequency signal. We used signal processing to analyze ECoG recordings from basic Wistar rats, and found that the delta rhythm modifies the gamma rhythm with a time delay of about $0.5 \mathrm{~s}$. The formation of gamma oscillations is triggered by increasing this low-frequency signal value, whereas reducing it causes gamma rhythm oscillation death, confirming the phase-amplitude link. These results came from the experimental data, not just for one channel, but all channels.

In addition, we constructed a model for gamma rhythm simulation based on the FHN model, fine-tuned its parameters. We demonstrated that it could regulate highfrequency signals such as gamma rhythm with delta rhythm as the system input.

In future studies, we plan to model the experiment on networks of biological neurons, inspired by the results of works [Dzhunusov and Fradkov, 2011], [Plotnikov and Fradkov, 2019]. Our plans also include the analysis of two noisy experiments using the developed tools.

\section{Acknowledgment}

Data analysis (Sections 3,4) is supported by the grant of Saint Petersburg State University (No. 84912397).

The model synthesis for simulation of gamma rhythm (Section 5) was performed in IPME RAS and supported by Ministry of Science and Higher Education of the Russian Federation (grant No. 075-15-2021-573).

\section{References}

Abubaker, M., Al Qasem, W., and Kvašňák, E. (2021). Working memory and cross-frequency coupling of neuronal oscillations. Frontiers in Psychology, 12, pp. 4506.

Berman, J. I., Liu, S., Bloy, L., Blaskey, L., Roberts, T. P., and Edgar, J. C. (2015). Alpha-to-gamma phaseamplitude coupling methods and application to autism spectrum disorder. Brain Connectivity, 5(2), pp. 8090. PMID: 25109843.

Bruns, A. and Eckhorn, R. (2004). Task-related coupling from high- to low-frequency signals among visual cortical areas in human subdural recordings. International Journal of Psychophysiology, 51 (2), pp. 97-116.

Buzsáki, G. (2010). Neural syntax: Cell assemblies, synapsembles, and readers. Neuron, 68 (3), pp. 362385.

Buzsáki, G. and Wang, X.-J. (2012). Mechanisms of gamma oscillations. Nature Reviews Neuroscience, $35(1)$, pp. 202-225. 
Canolty, R. and Knight, R. (2010). The functional role of cross-frequency coupling. Trends in Cognitive Sciences, 14 (11), pp. 506-515.

Canolty, R. T., Edwards, E., Dalal, S. S., Soltani, M., Nagarajan, S. S., Kirsch, H. E., Berger, M. S., Barbaro, N. M., and Knight, R. T. (2006). High gamma power is phase-locked to theta oscillations in human neocortex. Science, 313 (5793), pp. 1626-1628.

Carmichael, J., Gmaz, J., and van der Meer, M. (2017). Gamma oscillations in the rat ventral striatum originate in the piriform cortex. The Journal of Neuroscience, 37 (33), pp. 7962-7974.

Chuderski, A. (2016). Fluid intelligence and the crossfrequency coupling of neuronal oscillations. The Spanish Journal of Psychology, 19, pp. E91.

Clayton, M. S., Yeung, N., and Cohen Kadosh, R. (2018). The many characters of visual alpha oscillations. European Journal of Neuroscience, 48(7), pp. 2498-2508.

Dzhunusov, I. and Fradkov, A. (2011). Synchronization in networks of linear agents with output feedbacks. $A u$ tomation and Remote Control, 72, pp. 1615-1626.

Fell, J. and Axmacher, N. (2011). The role of phase synchronization in memory processes. Nature Reviews Neuroscience, 12 (2), pp. 105-118.

FitzHugh, R. (1961). Impulses and physiological states in theoretical models of nerve membrane. Biophysical Journal, 1 (6), pp. 445-466.

Folschweiller, S. and Sauer, J.-F. (2021). Respirationdriven brain oscillations in emotional cognition. Frontiers in Neural Circuits, 15.

Frolov, N. and Pitsik, E. (2021). Age-related changes in the brain functional connectivity during motor initiation. In Proc. SPIE 11847, Saratov Fall Meeting 2020: Computations and Data Analysis: from Molecular Processes to Brain Functions, p. 118470V.

Fu, C. M., Jiang, C., Chen, G. S., and Liu, Q. M. (2017). An adaptive differential evolution algorithm with an aging leader and challengers mechanism. applied soft computing. Applied Soft Computing, 57, pp. 60-73.

Furth, K. E., Mastwal, S., Wang, K. H., Buonanno, A., and Vullhorst, D. (2013). Dopamine, cognitive function, and gamma oscillations: role of D4 receptors. Front. Cell. Neurosci., 7, pp. 102.

Ghosn, N. J., Palmer, J. A., Borich, M. R., Ting, L. H., and Payne, A. M. (2020). Cortical beta oscillatory activity evoked during reactive balance recovery scales with perturbation difficulty and individual balance ability. Brain Sciences, 10 (11).

Gonzalez Burgos, G., Cho, R., and Lewis, D. (2015). Alterations in cortical network oscillations and parvalbumin neurons in schizophrenia. Biological Psychiatry, 77, pp. 1031-1040.

Gupta, K. (2019). Comparative study of transformations for time dependent signal and analysis of the earthquake motion dynamics using HHT. International Journal of Science and Research (IJSR), 8, pp. 900903.
Jensen, O. and Colgin, L. L. (2007). Cross-frequency coupling between neuronal oscillations. Trends in Cognitive Sciences, 11 (7), pp. 267-269.

Kiselev, A., Maksimenko, V., Shukovskiy, N., Pisarchik, A., Pitsik, E., and Hramov, A. (2019). Post-stroke rehabilitation with the help of brain-computer interface. In 2019 3rd School on Dynamics of Complex Networks and their Application in Intellectual Robotics (DCNAIR), pp. 83-85.

Köster, M., Finger, H., Graetz, S., Kater, M.-J., and Gruber, T. (2018). Theta-gamma coupling binds visual perceptual features in an associative memory task. Scientific Reports, $\mathbf{8}$.

Li, X., Fan, M., Sun, X., Li, Q., and Zhang, J. (2018). Research on the correlation of brain function based on improved phase locking value. Sheng wu yi xue gong cheng xue za zhi = Journal of biomedical engineering = Shengwu yixue gongchengxue zazhi, 35, pp. 350357.

Libelli, S. M. and Alba, P. (2000). Adaptive mutation in genetic algorithms. Soft Computing, 4 (2), pp. 76-80.

Mitra, S. K. (2001). Digital Signal Processing. 2nd Ed. McGraw-Hill, New York.

Mohamed, M. A., Yousem, D. M., Tekes, A., Browner, N., and Calhoun, V. D. (2004). Correlation between the amplitude of cortical activation and reaction time: A functional mri study. American Journal of Roentgenology, 183 (3), pp. 759-765. PMID: 15333368.

Moran, L. V. and Hong, L. E. (2011). High vs low frequency neural oscillations in schizophrenia. Schizophrenia Bulletin, 37 (4), pp. 659-663.

Nagumo, J., Arimoto, S., and Yoshizawa, S. (1962). An active pulse transmission line simulating nerve axon. Proceedings of the IRE, 50 (10), pp. 2061-2070.

Neske, G. T. (2016). The slow oscillation in cortical and thalamic networks: Mechanisms and functions. Frontiers in Neural Circuits, 9.

Osipova, D., Hermes, D., and Jensen, O. (2008). Gamma power is phase-locked to posterior alpha activity. PLoS ONE, 3 (12), pp. e3990.

Palva, J. M. (2005). Phase synchrony among neuronal oscillations in the human cortex. Journal of Neuroscience, 25 (15), pp. 3962-3972.

Panteley, E. and Loría, A. (2017). Synchronization and dynamic consensus of heterogeneous networked systems. IEEE Trans. Automat. Control, 62 (8), pp. 37583773.

Panter, P. (1965). Modulation, noise, and spectral analysis: applied to information transmission. McGrawHill, New York.

Papadimitriou, C., Vempala, S., Mitropolsky, D., Collins, M., and Maass, W. (2020). Brain computation by assemblies of neurons. Proceedings of the National Academy of Sciences of the United States of America, 117.

Park, H., Lee, D., Kang, E., Kang, H., Hahm, J., Kim, J., Chung, C. K., Jiang, H., Gross, J., and Jensen, O. 
(2016). Formation of visual memories controlled by gamma power phase-locked to alpha oscillations. Scientific Reports, 6, pp. 28092.

Pisarchik, A., Runnova, A., Maksimenko, V., Zhuravlev, M., Protasov, P., Kulanin, R., Khramova, M., and Hramov, A. (2018). Human personality reflects spatiotemporal and time-frequency eeg structure. PLOS ONE, 13, pp. e0197642.

Plotnikov, S. and Fradkov, A. (2019). On synchronization in heterogeneous fitzhugh-nagumo networks. Chaos Solitons \& Fractals, 121, pp. 85-91.

Rabiner, L. (1975). Theory and application of digital signal processing. Prentice-Hall, Englewood Cliffs, N.J.

Sauseng, P., Peylo, C., Biel, A. L., Friedrich, E. V. C., and Romberg-Taylor, C. (2019). Does cross-frequency phase coupling of oscillatory brain activity contribute to a better understanding of visual working memory? British Journal of Psychology, 110 (2), pp. 245-255.

Sevasteeva, E. S. and Plotnikov, S. A. (2021). A toolkit for a gamma rhythm processing and analysis. In Proc. $V$ Scientific School "Dynamics of Complex Networks and their Applications", pp. 182-185. 